\title{
Multi-label Problem Transformation Methods: a Case Study
}

\author{
Everton Alvares Cherman, Maria Carolina Monard, Jean Metz \\ Institute of Mathematics and Computer Science (ICMC) \\ University of São Paulo (USP) \\ Laboratory of Computational Intelligence (LABIC) \\ P.O. Box 668, Zip Code 13560-970 - São Carlos, SP, Brasil \\ \{echerman,mcmonard,metzz\}@icmc.usp.br
}

\begin{abstract}
Traditional classification algorithms consider learning problems that contain only one label, i.e., each example is associated with one single nominal target variable characterizing its property. However, the number of practical applications involving data with multiple target variables has increased. To learn from this sort of data, multi-label classification algorithms should be used. The task of learning from multi-label data can be addressed by methods that transform the multi-label classification problem into several single-label classification problems. In this work, two well known methods based on this approach are used, as well as a third method we propose to overcome some deficiencies of one of them, in a case study using textual data related to medical findings, which were structured using the bag-of-words approach. The experimental study using these three methods shows an improvement on the results obtained by our proposed multi-label classification method.
\end{abstract}

Keywords: machine learning, multi-label classification, binary relevance, label dependency.

\section{Introduction}

Traditional single-label classification methods are concerned with learning from a set of examples that are associated with a single label $y$ from a set of disjoint labels $L,|L|>1[9,1]$. However, there are several scenarios where each instance is labeled with more than one label at a time, i.e., each instance $\mathbf{x}_{i}$ is associated with a subset of labels $Y_{i} \subseteq L$. In this case, the classification task is called multi-label classification.

Multi-label classification has received increased attention in recent years, and has been used in several applications such as music categorization into emotions [8, 11], semantic images and video annotation [2, 18], bioinformatics [5, 15] and text mining [14], to mention just a few.

Existing methods for multi-label classification fall into two main categories, which are related to the way that single-label classification algorithms are used [13]:

1. problem transformation; and

2. algorithm adaptation.

Problem transformation methods map the multi-label learning task into one or more single-label learning tasks. When the problem is mapped into more than one single-label problem, the multi-label problem is decomposed into several independent binary classification problems, one for each label which participates in the multi-label problem. This method is called Binary Relevance $(B R)$. The final multi-label prediction for a new instance is determined by aggregating the classification results from all independent binary classifiers. Moreover, the multi-label problem can be transformed into one multi-class single-label learning problem, using as target values for the class attribute all unique existing subsets of multi-labels present in the training instances (the distinct subsets of labels). This method is called Label Power Set (LP). Any single-label learning algorithm can be used to generate the classifiers used by the problem transformation methods. Algorithm adaptation, on the other hand, extends specific learning algorithms in order to handle multi-label data directly.

The community has been working on the design of multi-label methods capable of handling the different relationships between labels, especially label dependency, co-occurrence and correlation. Some of these label relationships can be extracted from the training instances. In the LP method, for example, inter-relationships 
among labels are mapped directly from the data, since all the existing combinations of single-labels present in the training instances are used as a possible label in the correspondent multi-class single-label classification problem. In this context, the Binary Relevance method has been strongly criticized due to its incapacity of handling label dependency information [10]. In fact, the $B R$ method assumes that each single label is independent of the others, which makes it simple to implement and relatively efficient, although incapable of handling any kind of label relationship. Nevertheless, the Binary Relevance framework has several important aspects that help the development of new methods, which can be used to incorporate label dependency aiming to accurately predict label combination while keeping the simplicity and efficiency of the $B R$ framework.

In this work we use methods which belong to the problem transformation category: Label Power Set $(L P)$, which maps the multi-label problem into one single-label problem; Binary Relevance $(B R)$, which maps the multi-label problem into several single-label problems; and an extension of the $B R$ method, called $B R+($ BRplus $)$, initially presented in [3] and further improved in [4], which aims to overcome some of the limitations of $B R$. This work expands on our previous work [3] by using the improved version of $B R+$. A case study using textual data related to medical findings, which were structured using the bag-of-words approach, was carried out, showing an improvement in the results obtained by our proposed multi-label classification method.

The rest of this work is organized as follows: Section 2 describes multi-label classification, as well as the three problem transformation methods used in this work: $L P, B R$ and $B R+$. Section 3 defines the examplebased evaluation measures used to evaluate the experimental results. Section 4 describes the experimental set up and Section 5 reports the results. Section 6 concludes this work.

\section{Multi-label Classification}

Traditional supervised learning algorithms work under the single-label scenario, i.e., each example (instance) in the training set is associated with a single label characterizing its property. On the other hand, each example in the training set of multi-label learning algorithms is associated with multiple labels simultaneously, and the task is to predict the proper label set for unseen examples.

Let us formally define multi-label learning. Consider $D$ as the training set with $N$ examples $E_{i}=\left(\mathbf{x}_{i}, Y_{i}\right)$, where $i=1 . . N$. Each instance $E_{i}$ is associated with a feature vector $\mathbf{x}_{i}=\left(x_{i 1}, x_{i 2}, \ldots, x_{i M}\right)$ and a subset of labels $Y_{i} \subseteq L$, where $L=\left\{y_{j}: j=1 . . q\right\}$ is the set of $q$ possible labels. This representation is shown in Table 1. Considering this scenario, the task of a multi-label learning algorithm is to generate a classifier $H$ that, given an unlabeled instance $E=(\mathrm{x}, ?)$, is capable of accurately predicting its subset of labels $Y$, i.e., $H(E) \rightarrow Y$, where $Y$ is composed by the labels associated to the instance $E$.

Table 1: Multi-label data.

\begin{tabular}{|c|c|c|c|c|c|}
\hline & \multicolumn{4}{|c|}{$\mathbf{x}$} & $Y$ \\
\hline$E_{1}$ & $x_{11}$ & $x_{12}$ & $\ldots$ & $x_{1 M}$ & $Y_{1}$ \\
\hline$E_{2}$ & $x_{21}$ & $x_{22}$ & $\ldots$ & $x_{2 M}$ & $Y_{2}$ \\
\hline$\vdots$ & $\vdots$ & $\vdots$ & $\ddots$ & $\vdots$ & $\vdots$ \\
\hline$E_{N}$ & $x_{N 1}$ & $x_{N 2}$ & $\ldots$ & $x_{N M}$ & $Y_{N}$ \\
\hline
\end{tabular}

As stated earlier, in this work we use three multi-label methods which belong to the problem transformation category: Label Power Set $(L P)$, Binary Relevance $(B R)$, and $B R+($ BRplus), an extension of $B R$ we have proposed. It should be observed that methods from this category are algorithm independent. This is due to the fact that after transformation, any single-label learning algorithm can be used as a base-algorithm. A description of these three methods follows.

\subsection{Label Power Set}

This method transforms the multi-label problem into one single-label multi-class classification problem, where the possible values for the transformed class attribute is the set of distinct unique subsets of labels present in the original training data. Table 2 illustrates this idea, where the attribute space was omitted, since the transformation process only modifies the label space. The first table shows a multi-label set of instances in the original form, while the second table shows the same set of instances transformed into the Label Power Set format, where notation $y_{i, j, \ldots, k}$ means that the respective instance is labeled with the conjunction $y_{i} \wedge y_{j} \wedge \ldots \wedge y_{k}$. Observe that in the first table the set of labels $L$ is $\left\{y_{1}, y_{2}, y_{3}, y_{4}\right\}$ while after transformation, the set of multi-class labels is $\left\{y_{2,3}, y_{1,3,4}, y_{4}, y_{2,3}\right\}$.

Using this method, learning from multi-label examples corresponds to finding a mapping from the space of features to the space of label sets, i.e., the power set of all labels, resulting in up to $2^{|L|}$ transformed 
Table 2: Label Powerset multi-label dataset transformation example.

\begin{tabular}{c|l}
\cline { 2 - 2 } & \multicolumn{1}{|c}{$Y$} \\
\hline \hline$E_{1}$ & $Y_{1}=\left\{y_{2}, y_{3}\right\}$ \\
$E_{2}$ & $Y_{2}=\left\{y_{1}, y_{3}, y_{4}\right\}$ \\
$E_{3}$ & $Y_{3}=\left\{y_{4}\right\}$ \\
$E_{4}$ & $Y_{4}=\left\{y_{2}, y_{3}\right\}$ \\
\hline \hline
\end{tabular}

\begin{tabular}{c|c}
\cline { 2 - 2 } & $Y$ \\
\hline \hline$E_{1}$ & $y_{2,3}$ \\
$E_{2}$ & $y_{1,3,4}$ \\
$E_{3}$ & $y_{4}$ \\
$E_{4}$ & $y_{2,3}$ \\
\hline \hline
\end{tabular}

labels. Thus, although LP takes into account label dependency, when a large or even moderate number of labels are considered, the task of multi-class learning the label power sets would become rather challenging due to the tremendous (exponential) number of possible label sets. This is a drawback of the LP method. Another rather important issue is the class imbalance problem, which occurs when there are classes in the training set represented by very few examples. As the number of possible label sets increases with $|L|$, this is likely to occur.

\subsection{Binary Relevance}

The Binary Relevance method is a problem transformation strategy that decomposes a multi-label classification problem into several distinct single-label binary classification problems, one for each of the $q$ labels in the set $L=\left\{y_{1}, y_{2}, \ldots, y_{q}\right\}$. The $B R$ approach initially transforms the original multi-label training dataset into $q$ binary datasets $D_{y_{j}}, j=1 . . q$, where each $D_{y_{j}}$ contains all examples of the original multi-label dataset, but with a single positive or negative label related to the single label $y_{j}$ according to the true label subset associated with the example, i.e., positive if the label set contains label $y_{j}$ and negative otherwise. After the multi-label data has been transformed, a set of $q$ binary classifiers $H_{j}(E), j=1 . . q$ is constructed using the respective training dataset $D_{y_{j}}$. In other words, the $B R$ approach initially constructs a set of $q$ classifiers - Equation 1:

$$
H_{B R}=\left\{C_{y_{j}}\left(\left(\mathbf{x}, y_{j}\right)\right) \rightarrow y_{j}^{\prime} \in\{0,1\} \mid y_{j} \in L: j=1 . . q\right\}
$$

To illustrate the basic idea of the Binary Relevance transformation process, consider Table 3 which shows the four binary datasets constructed after the transformation ${ }^{1}$ of the multi-label dataset presented in Table 2. The possible values for the class attribute is "present" (or positive) or "not present" (or negative), denoted respectively by $y_{i}$ and $\neg y_{i}$.

Table 3: $B R$ transformation of the multi-label dataset shown in Table 2.

\begin{tabular}{c|c}
\cline { 2 - 2 } & $Y$ \\
\hline \hline$E_{1}$ & $\neg y_{1}$ \\
$E_{2}$ & $y_{1}$ \\
$E_{3}$ & $\neg y_{1}$ \\
$E_{4}$ & $\neg y_{1}$ \\
\hline \hline
\end{tabular}

\begin{tabular}{c|c}
\cline { 2 - 2 } & $Y$ \\
\hline \hline$E_{1}$ & $y_{2}$ \\
$E_{2}$ & $\neg y_{2}$ \\
$E_{3}$ & $\neg y_{2}$ \\
$E_{4}$ & $y_{2}$ \\
\hline \hline
\end{tabular}

\begin{tabular}{c|c}
\cline { 2 - 2 } & $Y$ \\
\hline \hline$E_{1}$ & $y_{3}$ \\
$E_{2}$ & $y_{3}$ \\
$E_{3}$ & $\neg y_{3}$ \\
$E_{4}$ & $y_{3}$ \\
\hline \hline
\end{tabular}

\begin{tabular}{c|c}
\cline { 2 - 2 } & $Y$ \\
\hline \hline$E_{1}$ & $\neg y_{4}$ \\
$E_{2}$ & $y_{4}$ \\
$E_{3}$ & $y_{4}$ \\
$E_{4}$ & $\neg y_{4}$ \\
\hline \hline
\end{tabular}

To classify a new multi-label instance, $B R$ outputs the aggregation of the labels positively predicted by all the independent binary classifiers.

A relevant advantage of the $B R$ approach is its low computational complexity compared with other multi-label methods. For a constant number of examples, $B R$ scales linearly with size $q$ of the label set $L$. Considering that the complexity of the base-classifiers is bound to $O(C)$, the complexity of $B R$ is $q \times O(C)$. Thus, the $B R$ approach is quite appropriate for not very large $q$. Nevertheless, as a large number of labels can be found in various domains, some divide-and-conquer methods have been proposed to organize labels into a tree-shaped hierarchy where it is possible to deal with a much smaller set of labels compared to $q$ [12].

However, the $B R$ method has a strong limitation regarding the use of label relationship information. As stated before, $B R$ fails to consider label dependency, as it makes the strong assumption of label independency. The $B R+$ method, explained next, attempts to diminish this limitation by trying to discover label dependency.

\footnotetext{
${ }^{1}$ As stated before, since only the label space is used during the transformation process, the attribute space is not shown.
} 


\section{$2.3 \quad B R+$}

It should be observed that $B R+$ does not make any attempt to discover label dependency in advance. The main idea of $B R+$ is to increment the feature space of the binary classifiers to let them discover by themselves existing label dependency.

In the training phase, $B R+$ works in a similar manner to $B R$, i.e., $q$ binary classifiers are generated, one for each label $y_{j} \in L$. However, there is a difference related to the $q$ binary datasets used to generate the binary classifiers. In $B R+$, the feature space of these datasets is incremented with $q-1$ features, which correspond to the other labels in the multi-label dataset. In other words, each $D_{y_{j}}$ binary training dataset is augmented with $\omega_{j}$ binary features where $\omega_{j}=L-\left\{y_{j}\right\}$. Figure 1, Training Phase, illustrates the binary datasets generated using a simple multi-label dataset with $L=\left\{y_{1}, y_{2}, y_{3}\right\}$. After this transformation, $B R+$ constructs a set of $q$ binary classifiers to classify new examples in this augmented feature space - Equation 2.

$$
H_{B R+}=\left\{C_{y_{j}}\left(\left(\mathbf{x} \cup \omega_{j}, y_{j}\right)\right) \rightarrow y_{j}^{\prime \prime} \in\{0,1\} \mid \omega_{j}=L-\left\{y_{j}\right\}, y_{j} \in L: j=1 . . q\right\}
$$

The classification of a new example could be carried out in a similar way to the standard $B R$ approach using these augmented binary classifiers. However, as each one of these classifiers has been generated using a feature space which is different to the original one, the unlabeled examples must also consider this augmented feature space. In other words, the feature space of the unlabeled examples must be augmented accordingly to the training set that generated the corresponding classifier.

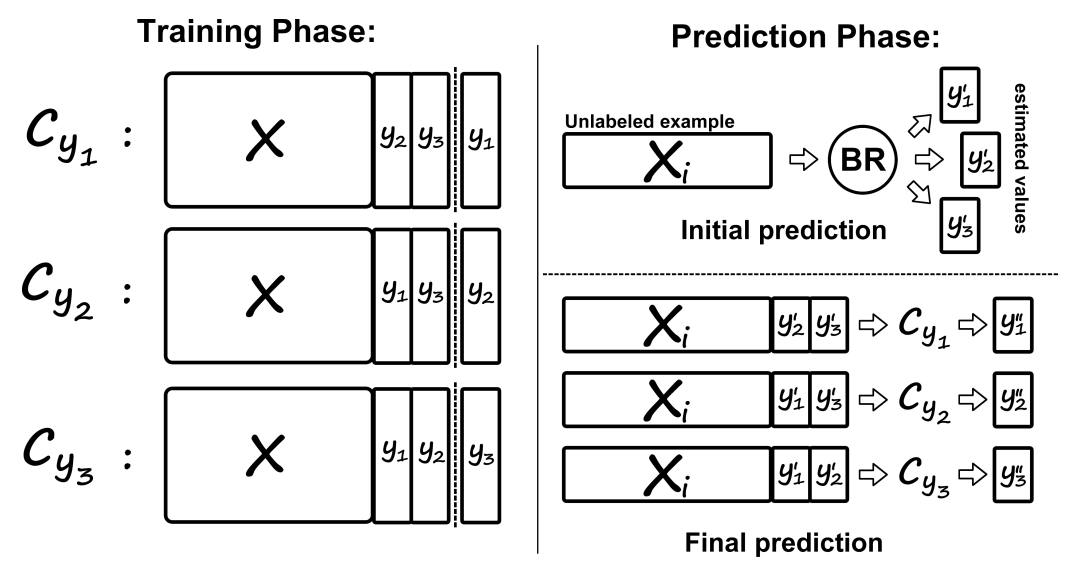

Figure 1: Illustration of $B R+$ considering a multi-label dataset with three labels $L=\left\{y_{1}, y_{2}, y_{3}\right\}$.

At this point, $B R+$ is faced with the following problem: The feature space of the unlabeled examples is different to the feature space of the training examples used to build the binary classifiers.

To solve this problem, it is necessary to apply the same transformation process used with the training examples to the unlabeled examples. To this end, the set of unlabeled examples is also replicated, resulting in $q$ unlabeled set of examples with the corresponding feature space for each of the binary classifiers generated in the training phase. On top of that, the value of those new features for the unlabeled examples are unknown, which leads to another problem:

How to estimate the values of the augmented features of unlabeled examples?.

In this paper, the values of the new features are estimated by $B R+$ in an intermediate step, where all the augmented feature values of unlabeled examples are initially predicted by $B R$ (Initial Prediction in Figure 1) considering the original training data. The values predicted by $B R$ are used by $B R+$ to complete the augmented feature space of the unlabeled examples. After this transformation, the unlabeled examples are classified by $B R+$ in a similar way to the standard $B R$ method, considering all the original features plus the augmented ones for each individual classifier. This prediction strategy, illustrated in Figure 1 (Final Prediction), is called $N U$ (No Update), since no modification is made to the initial estimates of the augmented features during the prediction phase.

Furthermore, the $B R+$ method can use a different strategy to predict the final set of labels for unlabeled examples, where the values of the initial prediction for the augmented features are updated with the prediction of the individual binary classifiers as the new predictions are obtained, considering a specific order of classification - Figure 2.

For example, if a predefined order to predict the individual labels is considered, each of the new values $y_{j}^{\prime \prime}$ can be used to update the previous value $y_{j}^{\prime}$ (initial prediction) of the corresponding augmented features of the unlabeled example. 


\section{Prediction Phase with Update Order:}

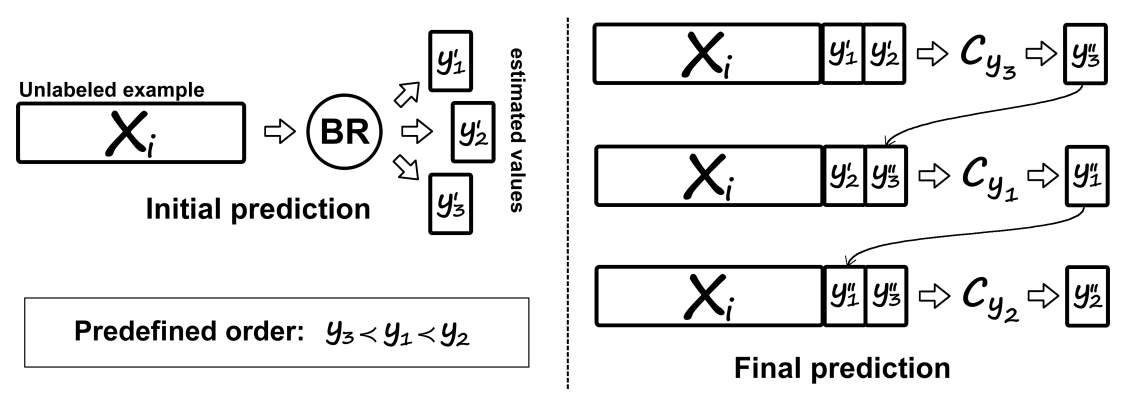

Figure 2: Illustration of $B R+$ update strategy using as predefined order $y_{3} \prec y_{1} \prec y_{2}$.

To illustrate, consider as a predefined order the frequency of single labels in the training set, where the least frequent labels are predicted first, and consequently, updated first. We call this ordering, which does not depend on the classifiers, Stat (Static Order). Another possible order takes into account the confidence of the initial prediction for each independent single label given by $B R$, where labels predicted with less confidence are predicted and updated first in the final prediction phase of $B R+$. We call this ordering $D y n$ (Dynamic Order). Figure 2 illustrates the update process considering a predefined order (Stat).

Regarding $B R+$ computational complexity, it can be observed that it simply duplicates $B R$ complexity. Thus, $B R+$ also scales linearly with the size $q$ of the label set $L$. Therefore, it is appropriate for a not very large $q$.

\section{Evaluation Measures}

Evaluation measures usually used in single-label classification tasks consider only two possible states for an instance regarding its classification: correct or incorrect. However, in multi-label tasks, an instance can be classified as partially correct. Therefore, multi-label classification problems require different metrics than the ones traditionally used in single-label classification. Some of the measures used in the multi-label context are adaptations of those used in the single-label context, while others were specifically defined for multi-label tasks. A complete discussion on the performance measures for multi-label classification is out of the scope of this paper, and can be found in [13]. In what follows, we show the measures used in this work to evaluate the three multi-label classification methods considered. These measures are based on the average differences of the true set $Y_{i}$ and the predicted set of labels $Z_{i}$, and are called example-based measures. The measures are Hamming Loss, Precision, Recall, F-Measure, Accuracy, and SubsetAccuracy, defined by Equations 3 to 8 respectively.

$$
\operatorname{Hamming} \operatorname{Loss}(H, D)=\frac{1}{N} \sum_{i=1}^{N} \frac{\left|Y_{i} \Delta Z_{i}\right|}{|L|}
$$

where $\Delta$ represents the symmetric difference between the set of true labels $Y_{i}$ and the set of predicted labels $Z_{i}$. When considering the Hamming Loss as the performance measure, the smaller the value, the better the algorithm performance is, with a lower bound equal to zero. For the next measures, greater values indicate better performance.

$$
\begin{gathered}
\operatorname{Precision}(H, D)=\frac{1}{N} \sum_{i=1}^{N} \frac{Y_{i} \cap Z_{i} \mid}{\left|Z_{i}\right|} \\
\operatorname{Recall}(H, D)=\frac{1}{N} \sum_{i=1}^{N} \frac{\left|Y_{i} \cap Z_{i}\right|}{\left|Y_{i}\right|} \\
F(H, D)=\frac{1}{N} \sum_{i=1}^{N} \frac{2\left|Y_{i} \cap Z_{i}\right|}{\left|Z_{i}\right|+\left|Y_{i}\right|} \\
\operatorname{Accuracy}(H, D)=\frac{1}{N} \sum_{i=1}^{N} \frac{\left|Y_{i} \cap Z_{i}\right|}{\left|Y_{i} \cup Z_{i}\right|} \\
\operatorname{SubsetAccuracy}(H, D)=\frac{1}{N} \sum_{i=1}^{N} I\left(Z_{i}=Y_{i}\right)
\end{gathered}
$$


where $I$ (true) $=1$ and $I$ (false) $=0$. It should be observed that SubsetAccuracy is a very strict evaluation measure as it requires an exact match of the predicted and the true set of labels.

\section{Experimental Set Up}

A case study was carried out using unstructured data of medical findings related to the Upper Digestive Endoscopy tests. It follows a description of the original data, as well as the pre-processing carried out to obtain the corresponding multi-label dataset.

\subsection{Case Study}

Gastroduodenal peptic diseases, such as ulcers and gastritis, characterize a frequent pathological entity in the population. Upper Digestive Endoscopy (UDE) is an important test for the diagnosis of these diseases. From this exam, a medical findings report with information about esophagus, stomach, duodenum and results of the exam is generated.

In this case study, a collection of 609 findings without patient identifications were used. These findings were collected by the Digestive Endoscopy Service of the Municipal Hospital of Paulinia, São Paulo state, Brazil, which were stored as plain text in digital media. Each medical finding consists of five sections: esophagus, stomach, duodenum, biopsy and conclusions (diagnosis) of the test. The first three sections, as well as the last one, are described in natural language (Portuguese), while the biopsy section is described by structured information.

In this work, stomach and duodenum information was used in order to predict the diagnosis related to these two sections. The information related to the diagnosis of the 609 findings were analyzed manually and mapped into five different classes, two directly related to the duodenum and three to the stomach. Thus, each finding is associated to one up to five labels. However, it is expected that the number of classes will increase after these findings are further analyzed by specialists.

Regarding the unstructured information of medical findings, it has been observed that medical findings present the two following characteristics:

1. the information is described using controlled vocabulary;

2. the information consists of simple assertive sentences.

These characteristics were explored in [6] to extract/construct relevant attributes in order to transform the non-structured information of medical findings, described in natural language (Portuguese), to the attributevalue format.

In this work, we use a simpler approach to transform the unstructured information of the stomach and duodenum medical findings into an attribute-value representation than the one used in [6]. To this end, we used PreTexT $2^{2}$, a locally-developed text pre-processing tool, which efficiently decomposes text into words (stems) using the bag-of-words approach. It also has facilities to reduce the dimensionality of the final representation using the well known Zipf's law and Luhn cut-offs, as well as by ignoring user defined stopwords [16]. PreTexT 2 is based on the Porter's stemming algorithm for the English language, which was adapted for Portuguese and Spanish.

In this work, we choose to represent terms as simple words (1-gram), which are represented by the stem (attribute name) of simple words. As attribute values we used the frequency of the correspondent stem. Moreover, the minimum Luhn cut-off was set to 6 (1\% of the number of medical findings), and the maximum Luhn cut-off was left unbounded. This way, the initial number of attributes, 244, was reduced to 108 attributes.

Summing up, the final attribute-value multi-label table, which represents the 604 medical findings used in the experiments, contains 108 attributes and $|L|=5$ labels.

\subsection{Algorithms}

$B R+$ was implemented using Mulan ${ }^{3}$, a package of Java classes for multi-label classification based on Weka ${ }^{4}$, a collection of machine learning algorithms for data mining tasks implemented in Java. $L P$ and $B R$ are already implemented in Mulan. Furthermore, four base-algorithms, implemented in Weka and described in [17], were used in the experiments: (i) J48 (Decision Tree); (ii) Naïve Bayes (NB); (iii) k-Nearest-Neighbor (KNN); (iv) SMO (Support Vector Machines).

\footnotetext{
${ }^{2}$ http://sites.labic.icmc.usp.br/pretext2/

${ }^{3}$ http://mulan. sourceforge.net

${ }^{4}$ http://www.cs.waikato.ac.nz/ml/weka/
} 
Preliminary experiments were carried out with $k=1,3,5,7,9$ to determine the most appropriate $k$ value for $K N N$. The best results were obtained with $k=3$, which was then used to execute $K N N$. Furthermore, the four base-algorithms were executed using default parameters.

\section{Experimental Results}

In this section, the performance of $B R+$ in the case study structured dataset is analyzed by means of comparison with $L P$ and $B R$. All the reported results were obtained using 10-fold cross validation with paired folds, i.e., the same training and testing partitions were used by $L P, B R$ and $B R+$ to obtain the average of the multi-label measures considered.

Table 4 shows the average of the example-based measure values described in Section 3. The numbers in brackets correspond to the standard deviation. For the sake of visualization, the best measure values are shown in bold. Arrow $\searrow$ indicates statistically significant degradation of $L P$ or $B R$ related to $B R+$. To this end, the Wilcoxon's signed rank test, a non-parametric statistical procedure for performing pairwise comparisons between two algorithms, was used.

Column $B R+$ TopLine shows the best possible results that could be obtained by $B R+$ using the corresponding base-algorithm. This value is obtained by a simple simulation which considers that $B R+$ always estimates the exact values of the augmented features of unlabeled examples during the Initial Prediction phase. In other words, the simulation considers $y_{i}^{\prime}=y_{i}$ - see Figure 1. Although we executed $B R+$ using the three different strategies $N U$, Stat and Dyn, we only show the results obtained using Dyn due to the fact that the three of them show similar results, and only in few cases $N U$ and Stat show a slight degradation compared to Dyn. These few cases are commented in the text.

Table 4: Average measure values for all base-algorithm. \ indicates statistically significant degradation of $L P$ ou $B R$ related to $\underline{B R+\text {. }}$

\begin{tabular}{|c|c|c|c|c|}
\hline & \multicolumn{4}{|c|}{$\mathrm{J} 48$} \\
\hline $\begin{array}{l}\text { H-Loss } \\
\text { Subset-Acc } \\
\text { Precision } \\
\text { Recall } \\
\text { F-Measure } \\
\text { Accuracy }\end{array}$ & $\begin{array}{l}\text { BR+TopLine } \\
0.02(0.01) \\
0.90(0.03) \\
0.96(0.02) \\
0.97(0.02) \\
0.96(0.02) \\
0.95(0.02) \\
\end{array}$ & $\begin{array}{l}B R+D y n \\
0.02(0.01) \\
0.90(0.03) \\
0.96(0.02) \\
0.97(0.02) \\
0.96(0.02) \\
0.95(0.02) \\
\end{array}$ & $\begin{array}{l}L P \\
0.04(0.01) \searrow \\
0.86(0.04) \\
0.94(0.02) \\
0.93(0.02) \searrow \\
0.93(0.02) \\
0.91(0.03) \searrow\end{array}$ & $\begin{array}{l}B R \\
0.03(0.01) \\
\mathbf{0 . 9 0 ( 0 . 0 3 )} \\
\mathbf{0 . 9 6 ( 0 . 0 2 )} \\
0.95(0.02) \\
0.95(0.02) \\
0.94(0.02) \\
\end{array}$ \\
\hline Accuracy & \multicolumn{4}{|c|}{$K N N$} \\
\hline $\begin{array}{l}\text { H-Loss } \\
\text { Subset-Acc } \\
\text { Precision } \\
\text { Recall } \\
\text { F-Measure } \\
\text { Accuracy } \\
\end{array}$ & $\begin{array}{l}\text { BR+ TopLine } \\
0.03(0.01) \\
0.88(0.04) \\
0.96(0.02) \\
0.95(0.02) \\
0.95(0.02) \\
0.93(0.03) \\
\end{array}$ & $\begin{array}{l}B R+D y n \\
0.04(0.01) \\
0.86(0.05) \\
0.95(0.02) \\
0.93(0.03) \\
0.94(0.03) \\
0.92(0.03) \\
\end{array}$ & $\begin{array}{l}L P \\
0.09(0.02) \searrow \\
0.75(0.04) \searrow \\
0.88(0.02) \searrow \\
0.85(0.02) \searrow \\
0.86(0.02) \searrow \\
0.83(0.03) \searrow \\
\end{array}$ & $\begin{array}{l}B R \\
0.08(0.02) \searrow \\
0.75(0.04) \searrow \\
0.89(0.03) \searrow \\
0.87(0.02) \searrow \\
0.87(0.02) \searrow \\
0.84(0.03) \searrow\end{array}$ \\
\hline Accuracy & \multicolumn{4}{|c|}{$\mathrm{SMO}$} \\
\hline $\begin{array}{l}\text { H-Loss } \\
\text { Subset-Acc } \\
\text { Precision } \\
\text { Recall } \\
\text { F-Measure } \\
\text { Accuracy }\end{array}$ & $\begin{array}{l}\text { BR+ TopLine } \\
0.02(0.01) \\
0.91(0.04) \\
0.97(0.02) \\
0.97(0.02) \\
0.96(0.02) \\
0.95(0.02)\end{array}$ & $\begin{array}{l}B R+D y n \\
0.02(0.01) \\
0.91(0.04) \\
0.96(0.02) \\
0.96(0.02) \\
0.96(0.02) \\
0.95(0.03) \\
\end{array}$ & $\begin{array}{l}L P \\
0.03(0.02) \\
0.88(0.05) \\
\mathbf{0 . 9 6 ( 0 . 0 3 )} \\
0.95(0.02) \\
0.94(0.03) \\
0.93(0.03) \\
\end{array}$ & $\begin{array}{l}B R \\
\mathbf{0 . 0 2 ( 0 . 0 1 )} \\
0.90(0.03) \\
0.95(0.02) \\
\mathbf{0 . 9 6 ( 0 . 0 2 )} \\
0.95(0.02) \\
0.94(0.02) \\
\end{array}$ \\
\hline Accuracy & \multicolumn{4}{|c|}{$\mathrm{NB}$} \\
\hline $\begin{array}{l}\text { H-Loss } \\
\text { Subset-Acc } \\
\text { Precision } \\
\text { Recall } \\
\text { F-Measure } \\
\text { Accuracy }\end{array}$ & $\begin{array}{l}\text { BR+ TopLine } \\
0.05(0.02) \\
0.79(0.07) \\
0.92(0.03) \\
0.96(0.02) \\
0.93(0.03) \\
0.90(0.03)\end{array}$ & $\begin{array}{l}B R+D y n \\
\mathbf{0 . 0 5}(\mathbf{0 . 0 2}) \\
0.79(0.07) \\
\mathbf{0 . 9 2}(\mathbf{0 . 0 3}) \\
\mathbf{0 . 9 5}(0.02) \\
\mathbf{0 . 9 3 ( 0 . 0 3 )} \\
\mathbf{0 . 9 0}(\mathbf{0 . 0 3})\end{array}$ & $\begin{array}{l}L P \\
\mathbf{0 . 0 5}(\mathbf{0 . 0 1}) \\
\mathbf{0 . 8 1}(\mathbf{0 . 0 4}) \\
0.91(0.04) \\
0.92(0.03) \searrow \\
0.91(0.03) \\
0.88(0.03)\end{array}$ & $\begin{array}{l}B R \\
\mathbf{0 . 0 5}(\mathbf{0 . 0 2}) \\
0.80(0.07) \\
\mathbf{0 . 9 2 ( 0 . 0 4 )} \\
0.93(0.03) \\
0.91(0.04) \\
0.89(0.05)\end{array}$ \\
\hline
\end{tabular}

It follows an analysis considering each of the four base-algorithm used in the experiments.

J48 The measure values for $B R+N U$ and $B R+$ Stat were the same as $B R+D y n$, except for the Accuracy measure which was equal to 0.94(0.02) for both of them, slightly less than the Accuracy of $B R+D y n$.

As can be observed, $B R+D y n$ was able to obtain the top line values of $B R+$ in all measures using $\mathrm{J} 48$ as a base-algorithm. However, $B R$, which does not attempt to discover label dependency, also shows good results. As J48 generates a decision tree (DT) classifier, it has the flexibility of choosing different subsets of features at different internal nodes of the tree such that the feature subset chosen optimally, according to some criterium, discriminates among the classes in that node. In other words, 
DTs have an embedded feature selection, and only a subset of features are considered in the nodes of the tree. Thus, unless the augmented features of unlabeled examples are in the classification tree, the classification tree fails to discover labels dependency.

Looking at the classification trees generated in each fold, it was observed that only in few cases the augmented features of unlabeled examples were part of the tree. This accounts for the slight improvement in some of the measures of $B R+$ over $B R$. It is worth observing that the participation of the augmented features of unlabeled examples in the tree classifier can be improved by attaching a positive weight to these features. This solution will be implemented and evaluated in the future.

Results using $L P$ show that for J48 as the base-algorithm it is not competitive with $B R+D y n$, and for three of the multi-label measures the results are significantly worse than $B R+D y n$.

KNN The measure values for $B R+N U$ and $B R+S t a t$ were the same as $B R+D y n$, except for the $\mathrm{F}$ Measure which was equal to $0.93(0.03)$ and $0.93(0.02)$ respectively, slightly less than the $\mathrm{F}$ Measure of $B R+$ Dyn.

Unlike J48, $K N N$ is a lazy learning algorithm which takes into account all feature values of the training set to classify an example. This characteristic enhances the properties of $B R+D y n$. As can be observed, all measures for $L P$ and $B R$ are significantly worse than for $B R+D y n$. Furthermore, all measure values obtained by $B R+D y n$ are near the $B R+$ TopLine values.

SMO The measure values for $B R+N U$ and $B R+S t a t$ were the same as $B R+D y n$.

SMO is a support vector machine algorithm. This family of algorithms manage to construct a decision boundary (maximum margin separator) with the largest possible distance to the example points. This sort of decision boundary helps them generalize well. For example, it can be observed that $L P$, although not better than $B R+D y n$, obtained its best results using SMO as the base-algorithm. As in the previous cases, $B R+D y n$ obtained the highest values for all measures, although none of them is significantly better.

NB Naïve Bayes uses Bayes theorem but does not take into account dependencies that may exist among features.

The measure values for $B R+D y n$ was the same for $B R+N U$ and $B R+S t a t$. Besides, BR+Dyn achieved the best result for all evaluation measures comparing with $B R$ and $L P$, except for Subset Accuracy. Furthermore, it outperformed LP with statistic significance for the Recall measure.

Summing up, as expected, it is possible to observe the influence of the base-algorithm in all methods tested. Nevertheless, in all cases $B R+D y n$ presented the best results, except the Subset Accuracy measure using NB as the base-algorithm. A point to consider is related to the difference of $B R$ and $B R+T o p L i n e$ evaluation measures, as greater differences indicate a higher possibility of $B R+$ improvements in relation to $B R$. This is the case when $K N N$ is used as the base-learning algorithm in which $B R+$ outperformed $B R$, as well as $L P$, with statistical significance.

\section{Conclusions and Future Work}

In this work, three different problem transformation methods, which map the multi-label learning problem into one or more single-label problems were considered: $L P, B R$ and $B R+$. The main objective of $B R+$, for us proposed and implemented, consists of exploring label dependency by only using the binary classifiers to discover and accurately predict label combinations. In this work, we used an improved implementation of $B R+$, where a simple simulation can be carried out to determine the best possible results that $B R+$ can achieve in a dataset, enabling the user to narrow the search for the best base-algorithm for any specific dataset using $B R+$.

A case study using unstructured Upper Digestive Endoscopy medical findings, which were structured using the bag-of-words approach, was conducted. More specifically, stomach and duodenum information was considered in order to predict the diagnosis related to these two sections. It should be observed that medical findings have some specific properties and most conclusions are multi-label.

Previous experimental results on several benchmark datasets have shown the potential of the $B R+$ approach to improve the multi-label classification performance in datasets which do not have a very high label space dimensionality, as is the case of medical findings.

In this work, we used a simple approach to structure the natural language information of the medical findings used in the case study. As future work, we plan to structure the information using a more elaborated approach to extract/construct relevant attributes from medical findings, as the one described in [7], where a 
specific method to support the construction of an attribute-value table from semi-structured medical findings is proposed, in order to compare the power of both approaches.

However, the performance of multi-label classification depends on many factors which are hard to isolate. As future work, we plan to investigate further label dependency looking at the problem from different perspectives, in order to theoretically understand the reasons for improvements of the different measures used in multi-label classification.

Acknowledgements: This research was supported by the Brazilian Research Councils FAPESP and CNPq.

\section{References}

[1] Ethem Alpaydin. Introduction to Machine Learning. The MIT Press, 2nd edition, 2010.

[2] Matthew R Boutell, Jiebo Luo, Xxipeng Shen, and Christopher M Brown. Learning multi-label scene classification. Pattern Recognition, 37:1757-1771, 2004.

[3] Everton Alvares Cherman, Jean Metz, and Maria Carolina Monard. Métodos multirrótulo independentes de algoritmo: um estudo de caso. In Proceedings of the XXXVI Conferencia Latinoamericana de Informática (CLEI), pages 1-14, Asuncion, Paraguay, 2010.

[4] Everton Alvares Cherman, Jean Metz, and Maria Carolina Monard. A simple approach to incorporate label dependency in multi-label classification. In Proceedings of the Mexican International Conference on Artificial Intelligence (MICAI'2010)- Advances in Soft Computing, volume 6438 of LNCC, pages 33-43, Pachuca(MX), 2010. Springer-Verlag.

[5] Amanda Clare and Ross D. King. Knowledge discovery in multi-label phenotype data. In Proceedings of the 5th European Conference on Principles of Data Mining and Knowledge Discovery, pages 42-53, London, UK, 2001. Springer-Verlag.

[6] Daniel De Faveri Honorato. Metodologia de transformação de laudos médicos não estruturados e estruturados em uma representação atributo-valor, 2008. Master dissertation. University of São Paulo, Brasil. http://www.teses.usp.br/teses/disponiveis/55/55134/tde-10062008-154826/publico/ dissertacaoDanielHonorato.pdf.

[7] Daniel Faveri Honorato, Maria Carolina Monard, Huei Diana Lee, and Wu Feng Chung. Uma abordagem de extração de terminología para construção de uma tabela atributo-valor a partir de documentos nãoestruturados. In Proceedings of the XXXIV Conferencia Latinoamericana de Informatica, pages 1-10. SADIO, 2008.

[8] Tao Li, Chengliang Zhang, and Shenghuo Zhu. Empirical studies on multi-label classification. Proceedings of the 18th IEEE International Conference on Tools with Artificial Intelligence (ICTAI'06), pages $86-92,2006$.

[9] Tom M. Mitchell. Machine Learning. McGraw-Hill Education, 1997.

[10] Jesse Read, Bernhard Pfahringer, Geoffrey Holmes, and Eibe Frank. Classifier chains for multi-label classification. In Wray L. Buntine, Marko Grobelnik, Dunja Mladenic, and John Shawe-Taylor, editors, Proceedings of the ECML/PKDD, volume 5782 of Lecture Notes in Computer Science, pages 254-269. Springer, 2009.

[11] K. Trohidis, G. Tsoumakas, G. Kalliris, and I. Vlahavas. Multilabel classification of music into emotions. In Proceedings of the 9th International Conference on Music Information Retrieval, pages 325-330, 2008.

[12] G. Tsoumakas, I. Katakis, and I. Vlahavas. Effective and efficient multilabel classification in domains with large number of labels. In Proceedings of the ECML/PKDD 2008 Workshop on Mining Multidimensional Data (MMD'08), 2008.

[13] G. Tsoumakas, I. Katakis, and I. Vlahavas. Mining multi-label data. Data Mining and Knowledge Discovery Handbook, pages 1-19, 2009.

[14] Naonori Ueda and Kazumi Saito. Parametric mixture models for multi-labeled text. In Suzanna Becker, Sebastian Thrun, and Klaus Obermayer, editors, Proceedings of the NIPS, pages 721-728. MIT Press, 2002 . 
[15] C. Vens, J. Struyf, L. Schietgat, S. Džeroski, and H. Blockeel. Decision trees for hierarchical multi-label classification. Machine Learning, 73(2):185-214, 2008.

[16] Sholom Weiss, Nitin Indurkhya, Tong Zhang, and Fred Damerau. Text Mining: Predictive Methods for Analyzing Unstructured Information. SpringerVerlag, 2004.

[17] Ian Witten, Eibe Frank, Geoffrey Holmes, and Mark Hall. Data Mining: Practical Machine Learning Tools and Techniques. Morgan Kaufman Publishers Inc., San Francisco, California, EUA, 3 edition, 2011.

[18] Min-ling Zhang and Zhi-hua Zhou. Multi-Label learning by instance differentiation. In Proceedings of the Twenty-Second AAAI Conference on Artificial Intelligence, pages 669-674, Vancouver - Canada, 2007. AAAI Press. 